Review

\title{
Differences in Clinical Characteristics and Treatments among Mycobacterial Osteomyelitis According to Species of Mycobacteria
}

\author{
${ }^{1}$ Asma Jafri, ${ }^{2}$ Luke DeLain and ${ }^{1}$ Tania del Rivero \\ ${ }^{1}$ Graduate Medical Education, Aventura Hospital and Medical Center, Aventura, FL, USA \\ ${ }^{2}$ Department of Family Medicine, St. Lucie Medical Center, Port St. Lucie, FL, USA
}

\author{
Article history \\ Received: 16-11-2018 \\ Revised: 05-11-2018 \\ Accepted: 16-01-2019 \\ Corresponding Author: \\ Asma Jafri \\ Graduate Medical Education, \\ Aventura Hospital and Medical \\ Center, Aventura, FL, USA \\ Email: Asma.Jafri@hcahealthcare.com
}

\begin{abstract}
In this review, cases of both Tuberculous Mycobacterial and Nontuberculous Mycobacterial osteomyelitis were analyzed to provide a concise understanding of the presentation and treatment of each reported Mycobacterial organism. Though rare, Mycobacterial osteomyelitis has continued to be a challenge in diagnosis and treatment due to its rarity as well as its indolent nature. As a result, it is usually diagnosed late in its course, with the patient having already experienced debilitating symptoms prior to diagnosis. In addition, another challenge of mycobacterial osteomyelitis is the specificity of treatment dependent on the mycobacteria causing infection; certain nuances in treatment can be the dividing line between a rapidly exacerbating infection and complete recovery. These nuances can be easily overlooked due to the fact that different types of mycobacterial osteomyelitis can present similarly, however requiring different forms of treatment. This manuscript will serve as a guide to various types of mycobacterial osteomyelitis classified by species of mycobacteria, outlining the preferred methods for diagnosis and most effective treatments for each.
\end{abstract}

Keywords: Osteomyelitis, Tuberculous Osteomyelitis, Non-Tuberculous Osteomyelitis, Mycobacteria

\section{Introduction}

Mycobacteria were first described as "fast-acid microorganisms" that could act as human pathogens by Pinner in 1935. For many years, the only known mycobacteria species were Mycobacterium tuberculosis, M. bovis and M.leprae (Wayne and Sramek, 1992). Since then, a wide array of mycobacteria species have emerged in part due to novel molecular biology techniques. Currently, there are 170 different species of Mycobacterium that have been documented and these different types of mycobacteria are more frequently being encountered in clinical settings (Forbes, 2017).

Mycobacterial infections are particularly difficult to treat due to the mycobacteria's unique cell wall and ability to evade the immune system. The cell wall is composed of a peptidoglycan layer, an arabinogalactan layer and a mycolic acid layer. The layers of the mycobacterial cell wall provide a distinct architectural integrity that results in low cell permeability and subsequently low success rate with antibiotics targeting the cell wall (Alderwick et al., 2015; Bhat et al., 2017). As a result, the majority of mycobacterial species are antibiotic resistant.

Another characteristic, which renders mycobacterial infections challenging, is the ability for mycobacteria to attack immune cells required for its clearance. Mycobacteria can infiltrate the intracellular space of macrophages and modify protein expression to harm these cells while remaining undetected by other components of the immune system (Glickman and Jr. Jacobs, 2001). TNF and other cytokines released by the immune system clear mycobacteria by promoting apoptosis of infected macrophages and other mechanisms. Therefore, patients who are immune-deficient or under anti-TNF therapy are more susceptible to mycobacteria infection (Hogan et al., 2017).

In this review, we will discuss both Tuberculous and Non-tuberculous mycobacterial osteomyelitis, different types of non-tuberculous mycobacteria that can cause osteomyelitis and the distinguishing clinical features and treatments for each. 


\section{Diagnosis of Mycobacterial Osteomyelitis}

Diagnosing mycobacterial infections is important because of the unique treatments required for mycobacterial species relative to other bacterial organisms (Hogan et al., 2017). The combination of clinical presentation, history of past exposure and laboratory and radiological findings help to raise suspicion for mycobacterial osteomyelitis. Routine laboratory work such as White Blood cell Count (WBC), Erythrocyte Sedimentation Rate (ESR) and C-Reactive Protein (CRP) can be used to establish an inflammatory pattern but are limited in value (Hogan et al., 2017). The WBC will often be normal, but the use of both ESR and CRP together greatly increases the sensitivity for osteomyelitis (Dunn and Husein, 2018). Blood cultures may be obtained but often take up to 6 weeks to come back positive for mycobacterium and in many cases they will come back negative (Dunn and Husein, 2018; Fritz and McDonald 2008). Nonetheless, they can help determine antibacterial susceptibility and help identify NonTuberculous Mycobacterium (NTM) (Hogan et al., 2017; Gardam and Lim, 2005).

Although imaging is not sufficient for diagnosis on its own, imaging modalities such as plain films, CT, ultrasound and MRI can be useful to support the diagnosis of mycobacterial osteomyelitis (Hogan et al., 2017). Radiographs are often used and can help differentiate mycobacterial osteomyelitis from other causes of osteomyelitis. For example, Pott's disease, a form of Mycobacterial Tuberculosis (MTB) spinal osteomyelitis, can be differentiated from pyogenic spinal osteomyelitis through characteristic features such as angular kyphosis, sparing of disc space and multilevel involvement of the vertebrae due to the infection spreading via the anterior longitudinal ligament (Hogan et al., 2017; Dunn and Husein, 2018). Ultrasound can be utilized to detect soft tissue damage and indicate the need for a biopsy. CT scans reveal bone deterioration and allow for earlier detection of the infection (Dunn and Husein, 2018). MRI is considered the best imaging modality for diagnosing mycobacterial osteomyelitis because it has the highest sensitivity and specificity (Dunn and Husein, 2018). MRI findings of a well-defined paraspinal abnormal signal and thin smooth enhancement of the abscess wall may be especially helpful in differentiating MTB infections from pyogenic infections involving the spine (Jung et al., 2004; Jain, 2010).

The Tuberculin Skin Test (TST) is a test that can help identify latent diseases but limited when testing for active diseases (Gardam and Lim, 2005). The TST lacks sensitivity and specificity especially in regards to immunocompromised patients and patients that have had the Bacillus Calmette-Guérin (BCG) vaccine (Choi et al., 2018). The TST is neither specific nor sensitive enough to make a diagnosis and a negative finding should not eliminate the diagnosis from the differential (Hogan et al., 2017).

Historically, the gold standard for diagnosis of mycobacterial osteomyelitis is a bone biopsy with cultures (Hogan et al., 2017; Fritz and McDonald, 2008). It is preferable to obtain tissue cultures versus peripheral blood cultures or cultures from pus because tissue cultures have a higher sensitivity (Hogan et al., 2017; Gardam and Lim, 2005). Although the presence of granulomas is not specific to mycobacterial infections, the histology may demonstrate necrotizing granulomas or reveal Acid Fast Bacillus (AFB) upon staining (Hogan et al., 2017; Gardam and Lim, 2005). The AFB smear test is often used to diagnose mycobacterial infections but other species of Nocardia can stain positive. A CT-guided aspiration is the diagnosis technique of choice; however, this can be difficult to perform with deep seated infections which are commonly found in mycobacterial osteomyelitis, so an open biopsy may be necessary (Murray et al., 2015). There is literature suggesting a combined reference standard including the clinical data, imaging and microbiology may be more appropriate considering the difficulty of diagnosing mycobacterial osteomyelitis (Norbis et al., 2014).

Polymerase Chain Reaction and other molecular tools may have the potential to become the best techniques for diagnosing mycobacterial osteomyelitis and assessing treatments; however, at this time the literature remains unclear on their effectiveness (Gardam and Lim, 2005). Interferon (IFN)- $\gamma$ Releasing Assays (IGRA's) have been used in the past but are unable to consistently differentiate between latent and active infections especially in mycobacterial epidemic areas (Hogan et al., 2017; Choi et al., 2018; Norbis et al., 2014). The enzymelinked immunosorbent assay (QuantiFERON-TB In-tube Test, QFT-GIT) has had reported sensitivities and specificities of $84 \%$ and $95 \%$; neither of which make the test diagnostic on its own (Dunn and Husein, 2018). Additionally Sunghim et al. (2018) reported the QFT-GIT to have a sensitivity of $91 \%$ and specificity of $50 \%$ in a retrospective study of 141 patients, further indicating that molecular biology tests are not accurate enough and a biopsy is needed to confirm the diagnosis.

\section{Mycobacterial Tuberculosis Osteomyelitis}

Osteomyelitis caused by Mycobacterium tuberculosis is indolent and insidious in its course and can take months to years to develop (Dunn and Husein, 2018). Patients may experience systemic signs similar to pulmonary tuberculosis such as fatigue, fevers, night sweats and weight loss; however, many patients' symptoms are localized only to the skeletal area affected (Hogan et al., 2017; Murray et al., 2015). While MTB osteomyelitis is most commonly 
associated with the spine, it can also occur in any skeletal area including proximal long bones, flat bones such as the sternum and tarsal bones (Shikhare et al., 2011). Patients with spinal tuberculosis, commonly referred to as Pott's disease, may have localized signs and symptoms upon presentation including back pain, paresthesias, radiculopathy and myelopathy as well as structure kyphotic deformity due to vertebral destruction (Hogan et al., 2017; Shikhare et al., 2011; Kaloostian and Gokaslan, 2013). In Pott's disease, MTB is most commonly localized in the anterior portion of the thoracolumbar spine (Hogan et al., 2017; Shikhare et al., 2011). Patients often have a spinal prominence known as a gibbus due to collapse of the vertebrae and associated kyphosis as well as prominent paraspinal abscesses that may cause additional spinal cord compression (Hogan et al., 2017; Dunn and Husein, 2018).

The management of mycobacterial tuberculosis generally consists of two stages of treatment recommended by the World Health Organization (WHO) (Rajasekaran and Khandelwal, 2013). The first phase is the intensive phase consisting of treatment with rifampin, isoniazid, pyrazinamide and ethambutol for 2 months followed by the continuation phase, which includes only rifampin and isoniazid for at least 4 months (Dunn and Husein, 2018; Varatharajahac et al., 2014; Rasouli et al., 2012). The total time of treatment varies according to the type of MTB infection (Rajasekaran and Khandelwal, 2013). The minimal treatment time regardless of the type of infection is 6-9 months and maximum treatment time can be up to 18 months (Shikhare et al., 2011; Murray et al., 2015). Specifically for spine MTB, the American Thoracic Society recommends 9 months of treatment with a 7month continuation phase instead of 4 months (Rasouli et al., 2012). To ensure the completion of medical therapy completion, the who also recommends a treatment strategy called Directly Observed Treatment Short-course (DOTS) that includes direct observation of the drug being swallowed 2-3 times a week (Dunn and Husein, 2018; Rajasekaran and Khandelwal, 2013). Infections from Multidrug-Resistant Tuberculosis (MDR-TB) organisms and extensively drugresistant tuberculosis (XDR-TB) are a growing concern. MDR-TB resistant to isoniazid and rifampin can result from incomplete treatment regimens and may require follow-up chemotherapy for up to 2 years using bactericidal antibiotics including fluoroquinolones and injectable aminoglycosides (Shikhare et al., 2011; Kumar, 2016). To combat the growing resistance, new drugs such as bedaquiline have been approved for MDR-TB although high cost and high toxicity have limited its use (Hoagland et al., 2016).

Surgical treatment for MTB in every skeletal area involves irrigation and debridement of abscess followed by potential bone grafting for areas of bone destroyed by the infection. Optimizing surgical debridement has been demonstrated to improve pharmacologic effectiveness (Varatharajahac et al., 2014). The indications for surgical treatment of spinal TB generally include neurological deficit, large paraspinal abscesses, spine instability due to kyphotic changes greater than 50-60 degrees, resistance to medical treatment and prevention of long-term complications such as paraplegia (Dunn and Husein, 2018; Murray et al., 2015; Varatharajahac et al., 2014; Rasouli et al., 2012; Kandwal and Jayaswal, 2016). There are several techniques for treating spinal TB including anterior debridement and decompression and fusion with bone grafts, posterior debridement and cord decompression with bone grafting, as well as combined anterior and posterior approaches with concomitant instrumentation and fusion (Murray et al., 2015; Kaloostian and Gokaslan, 2013; Rasouli et al., 2012). The principles of surgery including debridement, excision of the lesion and reconstruction and stabilization of the spine were first described in the 'Hong Kong Technique' and while debate exists as to which surgical approach is best used, these surgical goals remain the same (Murray et al., 2015).

\section{Non-Tuberculous Mycobacterial Osteomyelitis}

MTB is the most common cause of mycobacterial osteomyelitis, but many species of non-tuberculous mycobacteria (NTM) have also been found to cause osteomyelitis. Like most infectious diseases, patients who are most susceptible to developing MTB and NTM osteomyelitis are immunocompromised (HIV, corticosteroid therapy, receiving anti-TNF receptor antagonists, etc.) (Chan et al., 2001) and intravenous drug users (Talanow et al., 2013) but in NTM osteomyelitis, an event of penetrating trauma usually precedes the development of the infection. Prior to antiretroviral therapy, NTM osteomyelitis was mainly seen in HIV patients, but currently, it is usually seen in patients who are immunocompromised due to corticosteroid use. There have also been reported cases of NTM osteomyelitis in immunocompetent patients. Environmental reservoirs, especially water, were the most likely sources of infection, either via inhalation of contaminated aerosols or by invasion through the GI tract (Petitjean et al., 2004).

NTM is often misdiagnosed as MTB, but is resistant to anti-tuberculosis antibiotics due to the nature of its cell wall. An anti-tuberculous regimen in NTM osteomyelitis patients can lead to further bone deterioration, due to lack of appropriate treatment, which can possibly progress to paraplegia (Chan et al., 2001; Talanow et al., 2013). The main challenge with diagnosing NTM osteomyelitis is its inability to be detected early on in cultures or radiographies (Ehrlichman et al., 2015). When a tissue biopsy is collected, NTM will most likely appear with granulomas, whereas 
in MTB, the presence of granulomas is variable (Talanow et al., 2013). Non-Tuberculous Mycobacterial (NTM) infections often do not stain AFB, unlike MTB (Korres et al., 2007). CT scans of bones of patients with disseminated NTM will demonstrate abnormal thickening of the affected cortical bone with sclerotic changes, encroachment of the medullary cavity and chronic draining of the sinus tracts (Harp et al., 2018). If a granuloma is observed, no microorganism is detected by culture, PCR for MTB is negative and there is a lack of response to initial treatments, NTM should be suspected (Talanow et al., 2013).

DNA probes have been developed as additional tools to identify specific mycobacterial species such as $\mathrm{M}$. Avium, M. Intracellulare, M. Kansasii and other clinically relative species (Richter et al., 1999; Soini and Musser, 2001). These DNA probes hybridize with target rRNA sequences from the mycobacteria and have high degrees of sensitivity and specificity for certain species (Poonawala et al., 2017). For example, Richter et al. (1999) reported a sensitivity of $97.4 \%$ and specific of $100 \%$ for the identification of M. kansasii using a DNA probe, AccuProbe (Richter et al., 1999). However, there are also multiple drawbacks to DNA probes including lack of availability for every species of mycobacteria, inability to differentiate between members of the M. tuberculosis complex and misidentification of certain species (Poonawala et al., 2017; Soini and Musser, 2001).

NTM osteomyelitis typically involves the anterior inferior portion of the vertebral body and spreads under the anterior longitudinal ligament to involve the adjacent vertebrae (Chan et al., 2001). Similarly to MTB, the optimal treatment regimen would be surgical debridement with specified antimicrobial therapy (Kwan and Ho, 2010). Antimicrobial therapy is dependent on the organism and will be detailed below. Of note, in two cases in which an aminoglycoside was used, patients developed hearing loss and were switched to a macrolide (Morgan and Iseman, 1996). Below, the different types of NTM osteomyelitis organisms will be discussed, along with their typical presentations and effective treatments based on literature review.

\section{Mycobacterium Abscessus}

Mycobacterium Abscessus (M. Ab) is suspected to spread from the respiratory tract or cutaneous abrasions (Chan et al., 2001). The onset of the infection usually involves trauma to the bone or a puncture wound followed by a respiratory tract infection months later. After the respiratory illness resolves, patients tend to present with pain in the areas in which the trauma had occurred. It is unknown how $\mathrm{M}$. Ab propagates to a bone site (Chan et al., 2001). Treatment for $\mathrm{M}$. $\mathrm{Ab}$ is predominantly clarithromycin monotherapy for at least 6 months, however M. Ab subspecies should be identified prior to determining antibiotic treatment since some subspecies are more likely to develop resistance to macrolides. (Chan et al., 2001; Kim et al., 2016). The majority of $M$. abscessus subspecies abscessus and $M$. abscessus subspecies bolletii have inducible resistance to clarythromycin, while subspecies $M$. massiliense is susceptible to clarythromycin due to deletions in the erm (41) gene (Koh et al., 2011; Carvalho et al., 2018).

\section{Mycobacterium Avium}

Mycobacterium Avium (M. Av) is the most common NTM osteomyelitis and is also commonly found in HIV patients (Kim et al., 2016). In the cases of M. Av osteomyelitis, it was observed that the majority of patients would typically present with back pain, 4-6 months after experiencing trauma to the lumbar spine. In one case in which the patient presented for acute onset paraplegia and a one month history of back pain, there was no trauma observed, but the patient was a laborer intermittently for 10 years (Igram et al., 1997). Of note, in all the patients who were positive for M. Av osteomyelitis, caseation or progression to a psoas abscess was observed within the operative findings (Chan et al., 2001; Petitjean et al., 2004; Igram et al., 1997). Treatment involved surgical debridement and differing antibiotic regimens. The common factors among the regimens was a macrolide (clarithromycin or erythromycin) and ethambutol. Certain cases showed satisfactory results with the addition of a fluoroquinolone or Clofazimine. Treatment should be continued for 12-18 months for a favorable outcome (Chan et al., 2001; Igram et al., 1997). M. Avium subspecies paratuberculosis can survive in both aerobic and anaerobic conditions which allows for differential susceptibility to antibiotics (Parrish et al., 2017). Therefore combination therapies including both clarithromycin, an aerobically active drug and metronidazole, a highly effective drug for anaerobic infections, should be considered for this subspecies (Parrish et al., 2017; Lofmark et al., 2010).

\section{Mycobacterium Chelonae}

In all the cases of Mycobacterium Chelonae (M. $\mathrm{Ch}$ ) that were reviewed, the one characteristic that all the patients shared was the use of corticosteroid therapy. The reason for corticosteroid therapy varied from renal transplant (Petitjean et al., 2004) to SLE (Pruitt et al., 1993). Unlike other cases of NTM osteomyelitis, a traumatic event did not typically precede presentation of the infection and the time of presentation varied case by case. In most of the cases, patients presented with red nodulo-pustular skin lesions at the site of infection (Talanow et al., 2013; Korres et al., 2007; Harp et al., 2018). Due to the prevalence of these skin lesions in $\mathrm{M}$. Ch cases, it is suspected that the cause of the osteomyelitis is from 
disseminated cutaneous infection (Talanow et al., 2013). Another common characteristic amongst the cases was the presence of kyphosis with gibbus ((Talanow et al., 2013; Pruitt et al., 1993). In terms of diagnosis, various approaches were taken. These approaches include tissue cultures from the nodulo-pustular skin lesions (Korres et al., 2007; Harp et al., 2018), punch biopsy of the skin lesions followed by Ziehl-Neelsen staining, PCR and DNA sequencing (Talanow et al., 2013) and fine-needle aspiration biopsy of the bone lesion (Korres et al., 2007; Pruitt et al., 1993). Visualization and localization of the bone lesions were best done by CT scan of the bone (Talanow et al., 2013; Pruitt et al., 1993). MRI and plain radiographs were also utilized, but were not as accurate as CT. Treatment regimens varied among patients. In one patient, clarithromycin monotherapy was sufficient (Pruitt et al., 1993), but this is an unusual occurrence. Most cases had a multi-drug regiment including a macrolide, aminoglycoside and Imipinem. Along with those three, adding Ciprofloxacin, Linezolid, or Cilstatin led to recovery. Treatment should be implemented for at least 6 months.

\section{Mycobacterium Intracellulare}

Our literature search yielded only one case of Mycobacterium Intracellulare (M In.) that was reported in which a patient was receiving anti-cancer chemotherapy and radiation therapy to the thoracolumbar spine for 16 months prior to presentation (Kim et al., 2016). Diagnosis was done by needle biopsy of the infected tissue followed by hematoxylin eosin staining that showed granulomatous inflammation with necrosis (Kim et al., 2016). Cultures showed NTM which was specified to be $M$. In via sequencing of the 16S rRNA, rpo B and tuf gene (Kim et al., 2016). Effective treatment entailed surgical debridement followed by 24 months of Rifampin, ethambutol and clarithromycin. The patient showed full recovery without recurrence (Kim et al., 2016).

\section{Mycobacterium Marinum}

This mycobacteria is typically found in aquatic environments. Patients infected with Mycobacterium Marinum have often undergone a minor trauma in a region of the body that was later exposed to a contaminated body of water (Sivan et al., 2008; Clark et al., 1990; Nguyen et al., 2015). Since this mycobacterium thrives in cooler temperatures ranging from 30-32 degree $\mathrm{C}$, the superficial, cooler regions of the body tend to be affected more often, specifically the upper extremities (Sivan et al., 2008). Its presentation can take six weeks to six months to manifest and Mycobacterium Marinum infection should be suspected if the patient has negative bacterial tissue cultures, poor response to antibiotic treatments and a history of aquatic exposure (Sivan et al., 2008;
Nguyen et al., 2015). Diagnosis is confirmed through culture, but can be detected early via PCR from the biopsy specimen (Nguyen et al., 2015), using its specific probe (5'CACGGGATTCATGTCCTGT-3') (Portaels et al., 1997). Treatment involves a combination of surgical debridement and antibiotic therapies. The recommended combinations for antibiotic therapy are clarithromycin and/or rifampin plus ethambutol; clarithromycin and ciprofloxacin; or ethambutol and doxycycline (Sivan et al., 2008; Nguyen et al., 2015). In certain cases, chemotherapy has been added on to the treatment regimen for at least nine months (Clark et al., 1990; Nguyen et al., 2015).

\section{Mycobacterium Ulcerans}

Like other forms of NTM, Mycobacterium Ulcerans (M. Ulcerans), is commonly found in populations living near rivers, swamps, or wetlands (Pommelet et al., 2014) and it has been shown that one of the risk factors for bone involvement of $\mathrm{M}$. Ulcerans is lack of $\mathrm{BCG}$ vaccination (Portaels et al., 2004). A typical symptom of M. Ulcerans is the formation of skin lesions and the majority of patients with M. Ulcerans osteomyelitis have developed it from a contiguous infected skin lesion (Pommelet et al., 2014). Confirmation of diagnosis is done utilizing the $\mathrm{M}$. Ulcerans and M. Marinum specific probe (5'CACGGGATTCATGTCCTGT-3') (Portaels et al., 1997). The genetic characteristics that differentiate $M$. Ulcerans from M. Marinum are the presence of insertion sequences IS 2404 and IS 2606 (Stinear et al., 2000), as well as the hsp 65 gene (Roberts and Hirst, 1997). Treatment must include radical debridement (Pommelet et al., 2014; O'Brien et al., 2008) and an antibiotic regimen that varies depending on the case. Effective antibiotic regimens include 6 months of combined Rifampicin and ciprofloxacin (O'Brien et al., 2008) and Rifampin, ethambutol and co-trimoxazole for 2 months followed by clarithromycin and cotrimoxazole. Management includes adapted wound care and physiotherapy to prevent disability (Pommelet et al., 2014).

\section{Conclusion}

Mycobacterial osteomyelitis can be difficult to diagnose, specifically when it comes to identifying the species of mycobacteria involved, but this is crucial in determining the best treatment regimen (Table 1). Since NTM osteomyelitis is often misdiagnosed as MTB, it is important to discern their clinical presentations. Additionally, it is beneficial to differentiate between the various types of non-tuberculous mycobacteria causing osteomyelitis, since each requires a specific antibiotic regimen to maximize recovery. 
Table 1: Clinical presentation and treatment specific to each species of mycobacteria for osteomyelitis

Species of mycobacteria

Mycobacterium

Tuberculosis

Abscessus

Mycobacterium Avium

Mycobacterium

Chelonae

Mycobacterium Intracellulare

Mycobacterium

Marinum

Mycobacterium

Ulcerans
-Systemic signs may include fatigue,

fevers, night sweats and weight loss

-Most commonly associated with the spine,

but can affect any skeletal area

-Patients with spinal tuberculosis often present

with kyphosis with gibbus and paraspinal abcesses

that may cause spinal cord compression at spinal area of infection

-Onset often involves trauma to bone

or a puncture wound followed by a

respiratory tract infection months later

-Pain at the site of bone damage, even

after respiratory infection resolves

-Chronic back pain months after experiencing trauma to the lumbar spine

-Caseation which may progress to a psoas abscess

-Patients often present with red nodulo

-Pustular skin lesions at the site of infection- Patients often present with kyphosis with gibbus

-N/A due to insufficient cases reported in the literature

-Onset often involves a minor trauma

in a region of the body that is later

exposed to contaminated water

-Skin lesions which develop into skin

ulcerations spreading to the underlying bone
Antimicrobial therapy

-Rifampin, isoniazid, pyrazinamide and ethambutol treatment for 2 months, followed by continuation of rifampin and isoniazid treatment for another 4 months

-Total time of treatment varies with type of MTB infection

-Clarithromycin monotherapy for at least 6 months

-Antibiotic regimen which includes macrolide
(clarithromycin or erythromycin) and
ethambutol for 12-18 months
-Addition of fluoroquinolone or
Clofazimine may be favorable
-Corticosteroid therapy
-Multi-drug regimen including a macrolide,
aminoglycoside and Imipinem along with
ciprofloxacin, linezolid, or cilstatin
for at least 6 months.
-One case reported using rifampin, ethambutol
and clarithromycin for 24 months
-Clarithromycin and/or rifampin plus
ethambutol; clarithromycin and
ciprofloxacin; or ethambutol and doxycycline
-Chemotherapy may be added on to
the treatment regimen for at least nine months
-6 months of combined rifampin and
ciprofloxacin OR 2 months of rifampin,
ethambutol and co-trimoxazole followed by
clarithromycin and co-trimoxazole

\section{Author's Contributions}

Asma Jafri: Contributed to the conceptualization, writing the original draft, reviewing and editing.

Luke Delain: Contributed to writing the original draft, reviewing and editing.

Tania del Rivero: Contributed to project administration, writing the original draft, reviewing and editing.

\section{Ethics}

This article is original and contains unpublished material. The corresponding author confirms that all of the other authors have read and approved the manuscript and there are no ethical issues involved.

\section{References}

Alderwick, L.J.1., J.1. Harrison, G.S.1. Lloyd and H.L.1. Birch, 2015. The mycobacterial cell wallpeptidoglycan and arabinogalactan. Cold Spring Harb Perspect Med., 5: a021113.

DOI: $10.1101 /$ cshperspect.a021113.
Bhat, Z.S.1., M.A. Rather, M. Maqbool, H.U. Lah and S.K. Z. Yousuf et al., 2017. Cell wall: A versatile fountain of drug targets in Mycobacterium tuberculosis. Biomed. Pharmacother., 95: 1520-1534. DOI: 10.1016/j.biopha.2017.09.036

Carvalho, N.F.G., F. Pavan, D.N. Sato, C.Q.F. Leite and R.D. Arbeit et al., 2018. Genetic correlates of clarithromycin susceptibility among isolates of the Mycobacterium abscessus group and the potential clinical applicability of a PCR-based analysis of erm (41). J. Antimicrob. Chemother, 73: 862-866. DOI: 10.1093/jac/dkx476

Chan, E.D., K. Po-Marn, F. Kevin, P. D. Anthony and D.I. Michael, 2001. Vertebral osteomyelitis due to infection with nontuberculous Mycobacterium species after blunt trauma to the back: 3 examples of the principle of locus minoris resistentiae. Clin. Infect. Dis., 32: 1506-1510. DOI.org/10.1086/320155

Clark, R.B., H. Spector, D. Friedman, K.J. Oldrati and S.C. Nelson et al., 1990. Osteomyelitis and synovitis produced by Mycobacterium marinum in a fisherman. J. Clin. Microbiol., 28: 2570-2572. 
Choi, S., K.H. Jung, H.J. Son, S.H. Lee and J.M. Hong et al., 2018. Diagnostic usefulness of the QuantiFERON-TB gold in-tube test (QFT-GIT) for tuberculous vertebral osteomyelitis. Infect. Dis. (Lond), 50: 346-351.

DOI: $10.1080 / 23744235.2017 .1410282$

Dunn, R.N. and M.B. Husien, 2018. Spinal tuberculosis. Bone Joint J., 100: 425-431.

Ehrlichman, L.K., J.J.1. Kadzielski, E.P.1. Hyle and J.B.1. Jupiter, 2015. Nontuberculous mycobacterial osteomyelitis of the thumb: Successful treatment with serial debridement, antimicrobial therapy, external fixation and interphalangeal arthrodesis: A case report. J.B.J.S. Case Connect., 5: e87. DOI: $10.2106 /$ JBJS.CC.N.00245

Fritz, J.M. and J.R. McDonald, 2008. Osteomyelitis: Approach to diagnosis and treatment. Phys. Sportsmed, 36: nihpa116823. DOI: $10.3810 /$ psm.2008.12.11

Forbes, B.A., 2017. Mycobacterial taxonomy. J. Clin. Microbiol., 55: 380-383. DOI: 10.1128/JCM.01287-16

Glickman, M.S. and W.R. Jr. Jacobs, 2001. Microbial pathogenesis of Mycobacterium tuberculosis: Dawn of a discipline. Cell, 104: 477-485. DOI: 10.1016/S0092-8674(01)00236-7

Gardam, M. and S. Lim, 2005. Mycobacterial osteomyelitis and arthritis. Infect. Dis. Clin. North Am., 19: 819-30.

DOI: $10.1016 /$ j.idc.2005.07.008

Harp, G.M., R.D. Achcar and W.K. Drummond, 2018. Necrotizing osteomyelitis in a man with disseminated mycobacterium chelonae infection. IDCases, 12: 71-73.

DOI: 10.1016/j.idcr.2018.03.013.

Hoagland, D.T., D.T.1. Hoagland, J. Liu, R.B. Lee and R.E. Lee, 2016. New agents for the treatment of drug-resistant Mycobacterium tuberculosis. Adv. Drug Deliv. Rev., 102: 55-72.

DOI: $10.1016 /$ j.addr.2016.04.026

Hogan, J.I., R.M. Hurtado and S.B. Nelson, 2017. Mycobacterial Musculoskeletal Infections. Infect. Dis. Clin. North Am., 31: 369-382. DOI: $10.1016 /$ j.idc.2017.01.007

Igram, C.M., S.G. Petrie and M.B. Harris, 1997. Atypical mycobacterial vertebral osteomyelitis in an immunocompetent patient. Orthopedics, 20: 163-6. PMID: 9048393

Jain, A.K., 2010. Tuberculosis of the spine: A fresh look at an old disease. J. Bone Joint Surg. Br., 92: 905-13. DOI: 10.1302/0301-620X.92B7.24668

Jung, N.Y., W.H. Jee, K.Y. Ha, C.K. Park and J.Y. Byun, 2004. Discrimination of tuberculous spondylitis from pyogenic spondylitis on MRI. A.J.R. Am. J. Roentgenol., 182: 1405-1410.

DOI: 10.2214/ajr.182.6.1821405
Kaloostian, P.E. and Z.L. Gokaslan, 2013. Current management of spinal tuberculosis: A multimodal approach. World Neurosurg, 80: 64-5.

DOI: 10.1016/j.wneu.2013.01.120

Kim, C.J., U.J. Kim, H.B. Kim, S.W. Park and M.D. Oh et al., 2016. Vertebral osteomyelitis caused by non-tuberculous mycobacteria: Predisposing conditions and clinical characteristics of six cases and a review of 63 cases in the literature. Infect. Dis. (Lond), 48: 509-16.

DOI: $10.3109 / 23744235.2016 .1158418$

Koh, W.J., K. Jeon, N.Y. Lee, B.J. Kim and Y.H. Kook et al., 2011. Clinical significance of differentiation of Mycobacterium massiliense from Mycobacterium abscessus. A.m. J. Respir. Crit. Care Med., 183: 405-10. DOI: $10.1164 /$ rccm.201003-0395OC.

Korres, D.S., P.J. Papagelopoulos, K.A. Zahos, M.D. Kolia and G.G. Poulakou et al., 2007. Multifocal spinal and extra-spinal Mycobacterium chelonae osteomyelitis in a renal transplant recipient. Transpl. Infect Dis., 9: 62-5. DOI: 10.1111/j.1399-3062.2006.00178.x

Kumar, K., 2016. Spinal tuberculosis, natural history of disease, classifications and principles of management with historical perspective. Eur. J. Orthop. Surg. Traumatol., 26: 551-558.

Kwan, K. and S.T. Ho, 2010. Mycobacterium chelonae and Mycobacterium fortuitum infection following open fracture: A case report and review of the literature. Indian J. Med. Microbiol., 28: 248-50. DOI: $10.4103 / 0255-0857.66488$

Kandwal, P., G. Vijayaraghavan and A. Jayaswal, 2016. Management of tuberculous infection of the Spine. Asian Spine J., 10: 792-800. DOI: 10.4184 asj.2016.10.4.792

Lofmark, S., C. Edlund and C.E. Nord, 2010. Metronidazole is still the drug of choice for treatment of anaerobic infections. Clin. Infect. Dis., 50: S16-23. DOI: 10.1086/647939

Morgan, M.B. and M.D. Iseman, 1996. Mycobacterium bovis vertebral osteomyelitis as a complication of intravesical administration of Bacille CalmetteGuerin. Am. J. Med., 100: 372-373.

Murray, M.R., G.D. Schroeder and W.K. Hsu, 2015. Granulomatous vertebral osteomyelitis: An update. J. Am. Acad. Orthop. Surg., 23: 529-538. DOI: $10.5435 /$ JAAOS-D-13-00213

Nguyen, H.H., F. Nada, S.A. Muhammad and S.S. Dawd, 2015. Osteomyelitis infection of mycobacterium marinum: A case report and literature review. Case Rep. Infect. Dis., 2015: 905-920. DOI: $10.1155 / 2015 / 905920$ 
Norbis, L., R. Alagna, E. Tortoli, L.R. Codecasa and G.B. Migliori et al., 2014. Challenges and perspectives in the diagnosis of extrapulmonary tuberculosis. Expert. Rev. Anti. Infect. Ther., 12: 633-47. DOI: 10.1586/14787210.2014.899900

O'Brien, D.P., A. Eugene, H. Andrew and D.J. Paul, 2008. Successful treatment of Mycobacterium ulcerans osteomyelitis with minor surgical debridement and prolonged rifampicin and ciprofloxacin therapy: A case report. J. Med. Case Rep., 2: 123. DOI: 10.1186/1752-1947-2-123

Parrish, N., A. Vadlamudi and N. Goldberg, 2017. Anaerobic adaptation of Mycobacterium avium subspecies paratuberculosis in vitro: Similarities to M. tuberculosis and differential susceptibility to antibiotics. Gut. Pathog., 9: 34.

DOI: $10.1186 / \mathrm{s} 13099-017-0183-\mathrm{Z}$

Petitjean, G., U. Fluckiger, S. Scharen and G. Laifer, 2004. Vertebral osteomyelitis caused by nontuberculous mycobacteria. Clin. Microbiol. Infect., 10: $951-953$.

DOI: 10.1111/j.1469-0691.2004.00949.x

Pommelet, V., Q.B. Vincent, M.F. Ardant and A. Adeye, A. Tanase et al., 2014. Findings in patients from benin with osteomyelitis and polymerase chain reactionconfirmed Mycobacterium ulcerans infection. Clin. Infect. Dis., 59: 1256-1264. PMID: 25048846

Poonawala, H., E.P. Vincent, L. Lynn and C. Sheldon, 2017. Misidentification of mycobacterium paraense as mycobacterium avium complex by accuprobe. J. Clin. Microbiol., 55: 2283-2284. DOI: $10.1128 /$ JCM.00663-17

Portaels, F., M. Debacker, J. Aguiar and A. Guédénon, 2004. Mycobacterium bovis BCG vaccination as prophylaxis against mycobacterium ulcerans osteomyelitis in buruli ulcer disease. Infect. Immun., 72: 62-65. DOI: 10.1128/IAI.72.1.62-65.2004

Portaels, F.M., J. Agular, K. Fissette, P.A. Fonteyne and H. De Beenhouwer et al., 1997. Direct detection and identification of Mycobacterium ulcerans in clinical specimens by PCR and oligonucleotide-specific capture plate hybridization. J. Clin. Microbiol., 35: 1097-1100. PMID: 9114387
Pruitt, T.C., L.O. Hughes, R.D. Blasier, R.E. McCarthy and C.M. Glasier et al., 1993. Atypical mycobacterial vertebral osteomyelitis in a steroiddependent adolescent. A case report. Spine (Phila Pa 1976), 18: 2553-2555. PMID: 8303466

Rajasekaran, S. and G. Khandelwal, 2013. Drug therapy in spinal tuberculosis. Eur. Spine. J., 22: 587-593. DOI: $10.1007 / \mathrm{s} 00586-012-2337-5$

Richter, E., N. Stefan and H. Sven, 1999. Identification of Mycobacterium kansasii by using a DNA probe (AccuProbe) and molecular techniques. J. Clin. Microbiol., 37: 964-970.

Roberts, B. and R. Hirst, 1997. Immunomagnetic separation and PCR for detection of Mycobacterium ulcerans. J. Clin. Microbiol., 35: 2709-2711. PMID: 9316944

Rasouli, M.R., M. Mirkoohi, A.R. Vaccaro, K.K. Yarandi and V. Rahimi-Movaghar, 2012. Spinal tuberculosis: Diagnosis and management. Asian Spine J., 6: 294-308. DOI: https://doi.org/10.4184/asj.2012.6.4.294

Shikhare, S.N., S. Dinesh, R.S. Trishna and C.G.P. Wilfred, 2011. Tuberculous osteomyelitis and spondylodiscitis. Semin. Musculoskelet Radiol., 15: 446-458. DOI: 10.1055/s-0031-1293491

Sivan, M., B. Deepa, A. Nick and M.N. Martin, 2008. Mycobacterium marinum osteomyelitis of a long bone. Joint Bone Spine., 75: 600-602. DOI: 10.1016/j.jbspin.2007.08.011

Soini, H. and J.M. Musser, 2001. Molecular diagnosis of mycobacteria. Clin. Chem., 47: 809-814.

Stinear, T.P., A.J. Grant, D.R.J. Paul and J.K. Davies et al., 2000. Comparative genetic analysis of Mycobacterium ulcerans and Mycobacterium marinum reveals evidence of recent divergence. $\mathrm{J}$. Bacteriol., 182: 6322-30. PMID: 11053375

Talanow, R., H. Vieweg and R. Andresen, 2013. Atypical osteomyelitis caused by mycobacterium chelonae-A multimodal imaging approach. Case Rep. Infect. Dis., 2013: 528-795. DOI: $10.1155 / 2013 / 528795$

Varatharajahac, S., Y.P. Charlesac, X. Buybc, A. Walterac and J.P. Steibac, 2014. Update on the surgical management of Pott's disease. Orthop Traumatol. Surg. Res., 100: 229-35. DOI: 10.1016/j.otsr.2013.09.013

Wayne, L.G. and H.A. Sramek, 1992. Agents of newly recognized or infrequently encountered mycobacterial diseases. Clin. Microbiol. Rev., 5: 1-25. PMID: 1735092 